\title{
Neuropeptide Cotransmitters Released from an Identified Cholinergic Motor Neuron Modulate Neuromuscular Efficacy in Aplysia
}

\author{
Matthew D. Whim and Philip E. Lloyd \\ Department of Pharmacological and Physiological Sciences and Committee on Neurobiology, University of Chicago, \\ Chicago, Illinois 60637
}

Intrinsic buccal muscle 5 (15) in Aplysia is innervated by 2 motor neurons (termed B15 and B16). In addition to the classical transmitter ACh, B15 also contains the 2 neuropeptides $\mathrm{SCP}_{\mathrm{A}}$ and $\mathrm{SCP}_{\mathrm{B}}$. In a previous study, we demonstrated that the SCPs were released from the terminals of B15 in the 15 muscle and that this release was sufficient to raise CAMP levels in 15 muscle fibers. Significant peptide release occurred only when B15 was stimulated at high frequency or at lower frequencies with a relatively long burst duration (Whim and Lloyd, 1989). In the present article, we examine the possibility that the SCPs released from B15 modulate I5 muscle contractions produced by stimulation of the second motor neuron, B16. Application of exogenous SCPs to 15 muscles increased the amplitude and relaxation rate of $B 16$ evoked contractions. Stimulation of B15 using paradigms that have been shown previously to cause release of the SCPs resulted in a long-lasting increase in the amplitude and relaxation rate of muscle contractions evoked by B16. This modulation is unlikely to be due to the B15-induced muscle contractions themselves, because modulation of B16evoked contraction amplitude and relaxation rate was observed when the contractions were blocked transiently by a cholinergic antagonist during B15 stimulation. Conversely, stimulation of B15 at frequencies that produce no measurable release of the SCPs did not elicit significant modulation of B16-evoked contractions. The minimum B15 stimulation frequency required to elevate muscle CAMP levels or to modulate B16-evoked contractions was found be within the physiological range at which B15 fires during feeding. Therefore, the mechanism underlying the modulation of B16evoked contractions by $\mathbf{B 1 5}$ is likely to involve the release of the SCPs from B15 terminals in the 15 muscle. With respect to behavior, this modulation of muscle contractions would be most likely to occur during food-induced arousal when both motor nuerons fire at high frequency with brief interburst intervals.

\footnotetext{
Received Mar. 15, 1990; revised May 17, 1990; accepted May 24, 1990.

We thank P. J. Church, J. D. Hall, and W. L. Pearson for critical reading of the manuscript and C. White-Morris for expert technical assistance. This work was supported by NINCDS Grant NS 23596 and by Brain Research Foundation grants.

Correspondence should be addressed to Philip E. Lloyd, Department of Pharmacological and Physiological Sciences, University of Chicago, 947 East 58th Street, Chicago, IL 60637.

Copyright $(c) 1990$ Society for Neuroscience $0270-6474 / 90 / 103313-10 \$ 03.00 / 0$
}

Neuropeptides have been shown to be potent modulators of neuronal activity in many systems. In the majority of cases, the effects of the exogenous application of peptides were studied. Although this approach often leads to an unambiguous description of the effects of the peptides, it has certain limitations. A major problem is in assessing how similar the effects of the exogenous peptide application are to the effects of endogenous peptide release produced by direct neural stimulation. Clearly, an understanding of the parameters affecting the release of an endogenous peptide is needed before an estimate of its physiological role can be made.

In order to approach this problem, we have been studying the release of 2 neuropeptides from a model neuromuscular system in Aplysia. This system is bilaterally symmetrical and consists of identified motor neurons in the buccal ganglia that innervate buccal muscles involved in gencrating biting and swallowing movements. Each of the paired buccal ganglia contains 2 cholinergic motor neurons (B15 and B16; Cohen et al., 1978) that innervate intrinsic muscle 5 (I5; Howells, 1942; also called the ARC muscle; Cohen et al., 1978). In addition to ACh, both motor neurons also contain several modulatory neuropeptides. B15 contains members of the small cardioactive peptide (SCP) family and members of the buccalin family (Cropper et al., 1987a, 1988), while B 16 contains members of the buccalin family and the myomodulin family (Cropper et al., 1987b; Kupfermann et al., 1988). This and our previous study (Whim and Lloyd, 1989) have concentrated on the role of the SCPs in neuron B15. In Aplysia, the SCP family is made up of 2 peptides: $\mathrm{SCP}_{\mathrm{A}}\left(11\right.$ amino acids; Lloyd et al., 1987a) and $\mathrm{SCP}_{\mathrm{B}}(9$ amino acids; Morris et al., 1982), which have similar sequences and are processed from a single precursor (Mahon et al., 1985). There is considerable evidence that the SCPs serve a transmittcrlikc function in Aplysia (Abrams et al., 1984; Lloyd, 1986). They are located in dense-core vesicles in neurons and in varicosities in the CNS, gut, and 15 buccal muscle and are released from identified neurons in culture in a stimulation- and calciumdependent manner (Kreiner et al., 1986; Lloyd et al., 1986, 1988; Cropper et al., 1988; Reed et al., 1988).

There is substantial evidence that the SCPs function as neuromodulators at the I5 muscle. Both peptides have been shown to be synthesized by individually identified B 15 cell bodies and are known to be transported from the buccal ganglion to the I5 muscle, where immunoreactive fibers and varicosities ramify over the muscle fibers (Lloyd et al., 1984; Cropper et al., 1988; Lloyd, 1988). Furthermore, application of exogenous SCPs increases the amplitude and relaxation rate of motor-neuron-driven contractions, primarily via a cAMP-mediated enhancement 
of the excitation-contraction coupling mechanism (Weiss et al., 1979; Lloyd et al., 1984; Cropper et al., 1988).

Recently, 2 independent procedures were used to demonstrate that the SCPs were released from the terminals of B15 in I5 muscle (Whim and Lloyd, 1989). The first procedure indicated that intracellular stimulation of B 5 produced a depletion in the levels of the SCPs in the stimulated 15 muscle as compared to the unstimulated contralateral control muscle. The second procedure demonstrated that B15 stimulation caused marked increases in muscle cAMP levels. ACh and buccalin, the other transmitters known to be synthesized by B15, had no effects on cAMP levels in this muscle. Indeed, the only compounds present in I5 muscles that significantly increased cAMP levels were the SCPs and serotonin. However, the effects of the SCPs on muscle cAMP levels appear to be direct rather than involving a secondary release of serotonin because the magnitude of the effects was not reduced under conditions that inhibit transmitter release (Weiss et al., 1979; Lloyd et al., 1984; Whim and Lloyd, 1989). Both the depletion procedure and the elevation of CAMP indicated that the release of the SCPs from terminals of B15 in the muscle was sharply dependent on the stimulation parameters. Significant release of the SCPs only occurred when B15 was stimulated at a relatively high frequency with a long burst duration. Because B15 can produce cholinergically mediated contractions with stimulation parameters that would not cause measurable release of the SCPs, it appears that B15 can function in 2 states: as a predominantly cholinergic neuron or as a combined cholinergic/peptidergic neuron. Similar results describing the release of the SCPs from B 15 terminals in 15 muscle have recently been reported by Cropper et al. (1990).

The prescnt study describes a series of experiments designed to reveal the physiological roles of the SCPs that are released in 15 muscles by stimulation of B15. Because motor neurons B15 and B16 innervate the same I5 muscle fibers (Cohen et al., 1978 ) and the SCPs enhance muscle contractions primarily via a postsynaptic mechanism, we reasoned that it might be possible to reveal the role of the released SCPs by examining the effects of B15 stimulation on B16-evoked muscle contractions. Furthermore, this procedure would avoid possible complications from homosynaptic processes such as posttetanic potentiation (Cohen et al., 1978). In this study, we demonstrate that B15 stimulation enhances the amplitude and relaxation rate of B16evoked muscle contractions in a manner similar to that produced by the application of exogenous SCPs and that this enhancement occurs using stimulation parameters that previously have been shown to cause the release of the SCPs from B15 terminals.

\section{Materials and Methods}

Animals. Aplysia californica (100-200 gm) were obtained from Marinus Inc. (Long Beach, CA), maintained in circulating artificial sea water (ASW) at $16^{\circ} \mathrm{C}$, and fed dried seaweed every 3 days.

Measurement of 15 muscle contractions. Animals were immobilized with an injection of isotonic $\mathrm{MgCl}_{2}$. The dissection was carried out in high- $\mathrm{Mg}^{2+}(110 \mathrm{~mm})$, low-Ca ${ }^{2+}(2 \mathrm{~mm})$ ASW and was similar to that previously described (Whim and Lloyd, 1989). In brief, the buccal mass/ buccal ganglia complex was removed from the animal and bisected. One hemiganglion/hemibuccal mass was pinned out in a dish, and all nerves were severed except buccal nerve 3 . Muscles overlying I5 were removed, and one end of the 15 muscle was attached to an isotonic transducer (Harvard Apparatus). The ganglion was pinned to a small Sylgard platform and desheathed, and the preparation was superfused with normal ASW for $1-2 \mathrm{hr}$. Motor neurons B15 and B16 were identified by their position, size, and ability to produce contractions in I5 when stimulated. Typically, both cells were impaled with 2 electrodes (resistance, $\sim 5 \mathrm{M} \Omega$ ), one of which was used to inject current, while the other monitored membrane potential. A small tube was used to selectively superfuse the ganglion with high $-\mathrm{Mg}^{2+}(165 \mathrm{~mm})$, high-Ca ${ }^{2+}(33$ mM) ASW to suppress central synaptic activity (by raising the firing threshold of all neurons) while the bath was rapidly superfused with normal ASW. Individual spikes were driven by brief $(10 \mathrm{msec})$ depolarizing current pulses. In the majority of experiments, B16-induced contractions were evoked by a standard stimulation paradigm consisting of a 1-sec burst of stimuli at $20 \mathrm{~Hz}$ with an interburst interval of 100 sec. When the effects of stimulating B15 on B16-evoked muscle contractions were examined, B15 stimulation typically began $15 \mathrm{sec}$ after a B16 burst.

Compounds to be tested for their actions on motor-neuron-driven contractions were dissolved in ASW and applied via the superfusion. All chemicals were from Sigma, except myomodulin, $\mathrm{SCP}_{\mathrm{A}}$, and $\mathrm{SCP}_{\mathrm{B}}$ (Peninsula).

Measurement of $c A M P$. Measurement of CAMP was performed as previously described (Whim and Lloyd, 1989). Briefly, after stimulation in ASW (which in many experiments contained $0.5 \mathrm{~mm}$ hexamethonium chloride to block cholinergic contractions), I5 muscles were frozen with an electronic component freezer spray (GC-Electronics, Rockford, IL), then homogenized in $98 \%$ ethanol and $2 \% 2 \mathrm{~N} \mathrm{HCl}$ at $-30^{\circ} \mathrm{C}$ and centrifuged at $10,000 \times \mathrm{g}$. The supernatants were used for cAMP determinations using a commercial cAMP-binding protein assay (Amersham), while the pellets were used for protein determinations (Pierce Chemicals). Duplicate cAMP and protein determinations were carried out. To determine the effects of temperature on basal and $\mathrm{SCP}_{\mathbf{B}}$-stimulated cAMP levels, muscle segments were equilibrated at $16^{\circ} \mathrm{C}$ or $22^{\circ} \mathrm{C}$ in a water bath for $2 \mathrm{hr}$, incubated for $10 \mathrm{~min}$ in either $10^{-7} \mathrm{M} \mathrm{SCP}$ or ASW at the appropriate temperature, then processed as described above. Each of the paircd I5 muscles was bisceted along its long axis so that all 4 incubation conditions could be carried out on muscle segments from the same animal.

\section{Results}

\section{Modulation of I5 muscle contractions by exogenous} neuropeptides

The amplitude of the muscle contractions induced by stimulation of motor neuron $\mathrm{B} 16$ was reversibly increased by the application of the SCPs in the superfusate (Fig. 1A). Furthermore, the peptides also produced a pronounced increase in the muscle relaxation rate (Fig. $1 B$ ). In these experiments, we also confirmed a previous report that increasing the contraction amplitude by increasing B16 firing frequency leads to a small decrease in the relaxation rate (Cropper et al., 1988). Therefore, increased relaxation rates are not causally or necessarily linked to increased contraction amplitude. Over the range from $10^{-9}$ to $10^{-6} \mathrm{M}$, myomodulin also potentiated both contraction amplitude and muscle relaxation rate in a manner similar to the SCPs (Fig. 2). Myomodulin and the SCPs also had similar effects on B15-cvoked contractions.

\section{Stimulation of B15 modulates B16-evoked muscle contractions}

We next examined the effect of B15 stimulation on the contractions evoked by stimulation of $\mathrm{B} 16$ at $20 \mathrm{~Hz}$ for $1 \mathrm{sec}$ with an interburst interval of $100 \mathrm{sec}$. When B15 was stimulated at $20 \mathrm{~Hz}$ (4-sec bursts with an interburst period of $6 \mathrm{sec}$ for 60 $\mathrm{sec}$ ) during the interval between successive B16 contractions, the subsequent B16-evoked contractions increased in amplitude (Fig. 3). Examination of superimposed B16 contractions revealed that there were also marked increases in muscle relaxation rate after $B 15$ stimulation. Both the increases in contraction amplitude and relaxation rate reversed slowly over a period of 1-2 hr. The magnitude of the increase in B16-evoked contraction amplitude could be increased by stimulating $\mathrm{B} 15$ at higher frequencies or in bursts of longer duration. Figure $3 C$ 


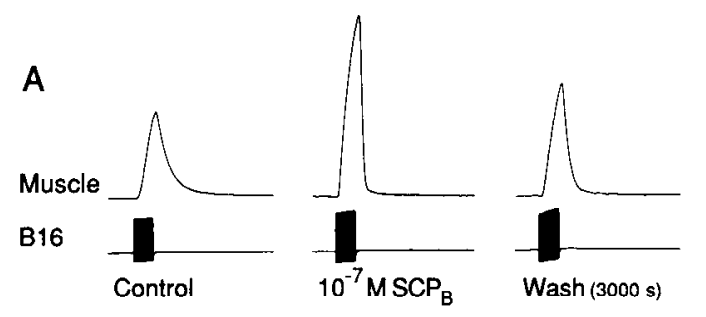

B

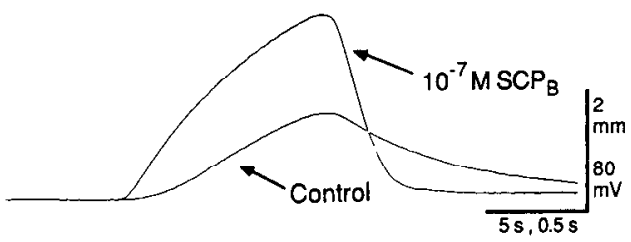

Figure 1. Modulation of B16-evoked I5 muscle contractions by superfusion with $\mathrm{SCP}_{\mathrm{B}}, A$, Amplitudes of $\mathrm{I} 5$ muscle contractions produced by bursts of action potentials in B16 were reversibly increased by superfusion with $10^{-7} \mathrm{M} \mathrm{SCP}_{\mathrm{B}}$. Contractions were evoked by stimulation of B16 at $20 \mathrm{~Hz}$ for $1 \mathrm{sec}$ with $100-\mathrm{sec}$ interburst intervals. $B$, Examination of the B16-induced muscle contractions at a faster chart speed before and at the peak of the response to $\mathrm{SCP}_{\mathrm{B}}$. In addition to the increase in the amplitude, there was also a large increase in the muscle relaxation rate produced by $\mathrm{SCP}_{\mathrm{B}}$. In this and following figures, the expanded traces of muscle contractions were aligned so that the motor neuron bursts that evoked the contractions were superimposed. In all experiments, central neurons were not exposed to the solutions bathing the muscle because the ganglia were superfused independently with high- $\mathrm{Mg}^{2+}$, high-Ca ${ }^{2+}$ ASW (see Materials and Methods).

shows the effects of stimulating B 15 at different frequencies with a fixed burst duration and interburst interval.

The mechanism underlying this increased contraction amplitude could involve an increase in the amplitude of the compound EJP or a change in the efficiency of excitation coupling in the muscle fibers. To differentiate between these possibilities, the effects of stimulation of B15 on B16-evoked contractions were determined in a split-muscle preparation in which contractions were measured in one half of the muscle while B16evoked compound EJPs were simultaneously recorded by a suction electrode placed on the other half of the muscle. The suction electrode was used because intracellular impalements could not be held during the tetanic stimulation of B15. Great care was taken to position the suction electrode so that the seal on the muscle was not compromised by the evoked contractions. To produce large individual EJPs, B16 was fired at a relatively low frequency $(8 \mathrm{~Hz})$ in these experiments. Stimulation of B15 potentiated the amplitude of the B16-evoked contractions with no increase in the compound EJPs (Fig. 4). Application of the SCPs to the I5 muscle also modulates I5 contractions with little or no effect on EJPs. Thus, stimulation of B15 and application of the SCPs both increase the efficiency of excitation-contraction coupling in the $\mathrm{I} 5$ muscle.

In a previous study, we demonstrated that the release of the SCPs from B15 terminals, as measured by depletion of the SCPs from stimulated muscle or by elevation of muscle cAMP levels, was dependent on the pattern of B15 stimulation (Whim and Lloyd, 1989). In that study, 3 patterns of stimulation, each containing the same overall number of spikes, were tested. These stimulation paradigms were $5 \mathrm{~Hz}$ tonic, $25 \mathrm{~Hz}$ for $2 \mathrm{sec}$ with 8-sec interburst intervals, and $50 \mathrm{~Hz}$ for $1 \mathrm{sec}$ with 9 -sec inter-
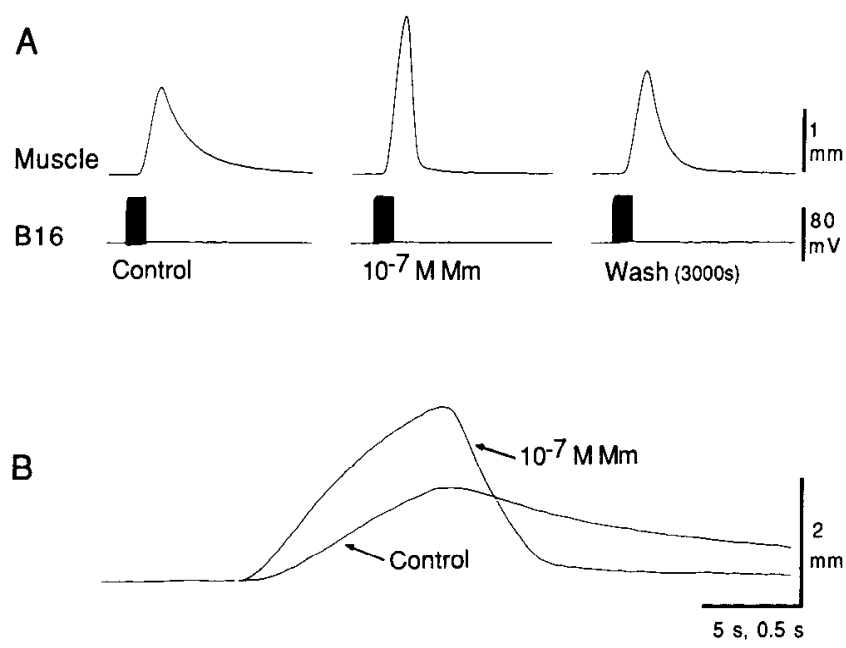

Figure 2. Modulation of B16-evoked I5 muscle contractions by superfusion with myomodulin. $A$, Amplitudes of 15 muscle contractions produced by bursts of action potentials in $\mathrm{B} 16$ were reversibly increased by superfusion with $10^{-7} \mathrm{M}$ myomodulin $(\mathrm{Mm})$. Contractions were evoked by stimulation of $\mathrm{B} 16$ at $20 \mathrm{~Hz}$ for $1 \mathrm{sec}$ with 100 -sec interburst intervals. $B$, Examination of the B16-induced muscle contractions at a faster chart speed before and at the peak of the response to myomodulin. Myomodulin increased the amplitude and rate of relaxation of muscle contractions in a manner similar to the SCPS.

vals. Only the last paradigm produced depletion of the SCPs and elevation of muscle cAMP levels. We wished to determine whether the modulation of B16-evoked contractions by B15 stimulation showed the same dependence on the pattern of stimulation. In Figure 5, B15 was stimulated for $70 \mathrm{sec}$ with each of the patterns described above. The tonic $5-\mathrm{Hz}$ pattern actually produced a transient decrease in B16-evoked contractions, a phenomenon for which we have no explanation. The $25-\mathrm{Hz}$, 2 -sec paradigm produced a small, brief enhancement, while the $50-\mathrm{Hz}, 1-\mathrm{sec}$ paradigm produced a larger and long-lasting increase in B16-evoked contractions. Thus, these observations parallel our previous results on the effects of stimulation pattern on release of the SCPs.

\section{B15-dependent modulation of B16-evoked contractions does not require cholinergic transmission during $B 15$ stimulation}

One possible mechanism for the enhancement of B16-evoked contractions by B 15 stimulation might be a change in the properties of the muscle rclated to the large contractions produced by $\mathrm{B} 15$ stimulation. However, 2 lines of evidence indicate that this mechanism is unlikely. First, stimulation of B15 using paradigms that result in contractions that are smaller than the "control" B16-evoked contractions still enhances the subsequent B16-evoked contractions (data not shown). Second, if muscle contractions were blocked by the application of a cholinergic antagonist (hexamethonium) before B15 was stimulated, then the B16-evoked contractions, which gradually returned as the antagonist was washed from the bath, were enhanced in both amplitude and relaxation rate (Fig. 6). Control experiments that tested the effects of the antagonist on B16-evoked contractions in the absence of B15 stimulation indicated that hexamethonium itself had no potentiating effect on either amplitude or relaxation rate. These results demonstrate that the B15-induced muscle contractions themselves are not likely to be responsible 


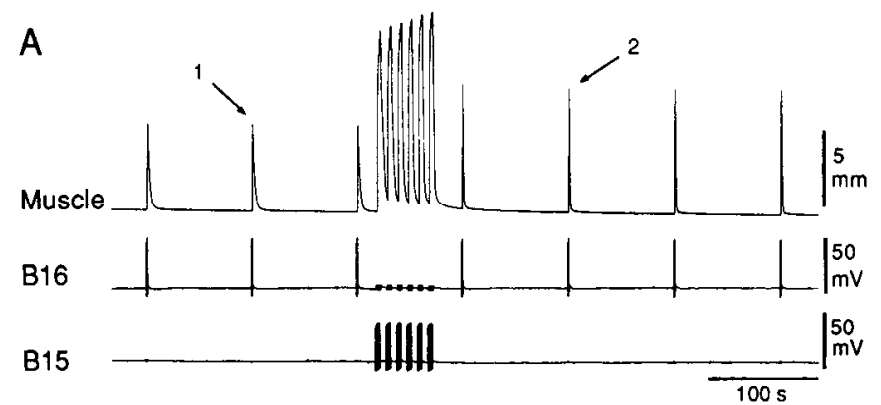

B

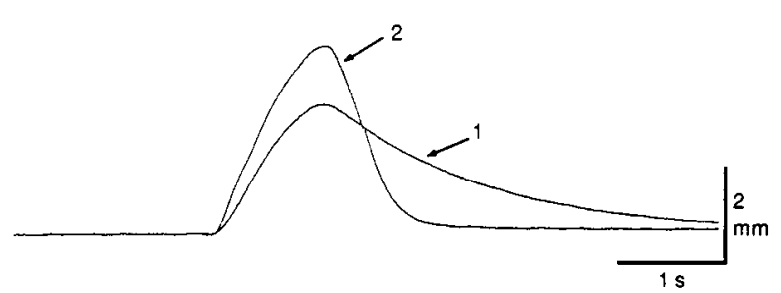

C

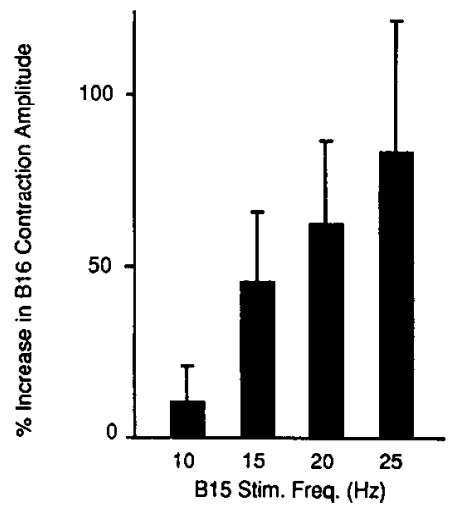

Figure 3. Modulation of B16-evoked I5 muscle contractions by stimulation of B15. A, Stimulation of B16 at $20 \mathrm{~Hz}$ for $1 \mathrm{sec}$ with $100-\mathrm{sec}$ interburst intervals elicited reproducible 15 muscle contractions. A period of B 15 stimulation ( $25 \mathrm{~Hz}$ for 4 -sec burst with an interburst interval of $6 \mathrm{sec}$ for $60 \mathrm{sec}$ ) was then interposed between successive B16 bursts. The subsequent I5 muscle contractions evoked by stimulation of B16 were increased in amplitude. In this and subsequent figures, capacitive coupling was often observed between current and voltage electrodes. $B$, Examination of B16-evoked muscle contractions $(A, 1,2)$ on an expanded time scale illustrates that, in addition to the increase in contraction amplitude, there was also an increase in the muscle relaxation rate. $C$, Effect of stimulating $\mathrm{B} 15$ on $\mathrm{I} 5$ muscle contractions produced by stimulation of B16 as described above. B15 was stimulated at the indicated frequencies in 4-sec bursts with 6-sec interburst intervals for $45 \mathrm{sec}$. Values compare mean \pm SEM of 3 Bl6-evoked contractions just before and after $B 15$ stimulation in 4 preparations.

for the potentiated B16-evoked contractions seen following B15 stimulation.

\section{Kinetics of increased muscle cAMP levels produced by $B 15$ stimulation}

Previous work has demonstrated that application of exogenous SCPs to I5 muscles produces a dose-dependent elevation of muscle cAMP levels and that stimulation of B15 for $10 \mathrm{~min}$ with appropriate paradigms also produced significant increases in cAMP in the muscle (Lloyd et al., 1984; Whim and Lloyd, 1989). Because the stimulation periods used in this study were shorter than $10 \mathrm{~min}$, we analyzed the time course of the increase

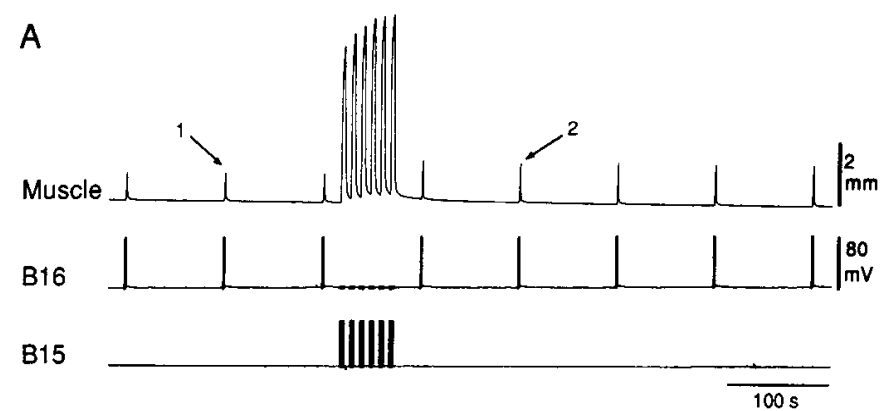

B

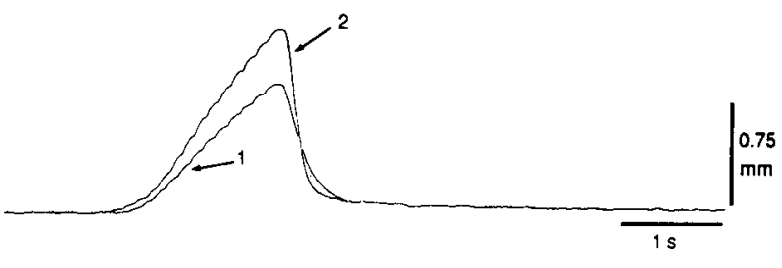

C

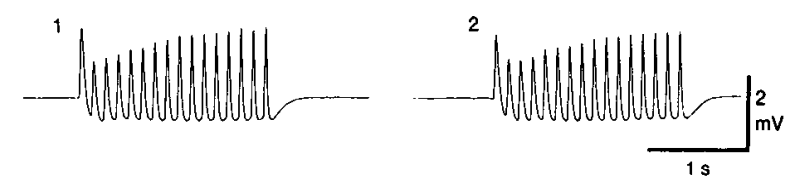

Figure 4. Modulation of B16-evoked muscle contractions by stimulation of B15 occurred via increase in excitation-contraction coupling. $A$, I5 muscle contractions evoked by stimulation of B16 $(8 \mathrm{~Hz}$ for $2 \mathrm{sec}$ with interburst intervals of $100 \mathrm{sec}$ ) were potentiated when B15 was stimulated $(25 \mathrm{~Hz}$ for $4 \mathrm{sec}$ with 6-sec intervals for $60 \mathrm{sec})$ between successive $\mathrm{B} 16$ bursts. $B$, Examination of B16-induced muscle contractions on an expanded time scale before $(I)$ and after $(2)$ B 15 stimulation illustrates the increased relaxation rate. At this low rate of B16 stimulation, each EJP produces small individual contractions that are visible as small steps on the rising phase of the contractions. $C$, Examination of the B16-evoked compound EJPs recorded in the I5 muscle before (I) and after (2) B15 stimulation. Although there was potentiation of I5 muscle contractions, there was no increase in the amplitude of the compound EJPs. $C, 1$ and 2, were associated with B16-evoked muscle contractions $B, l$ and 2 , respectively.

in cAMP produced by B15 activity. Significant elevations of cAMP were apparent after only $1 \mathrm{~min}$ of stimulation (Fig. 7). Maximal levels were observed by $10 \mathrm{~min}$, and these levels were maintained for $30 \mathrm{~min}$, with a moderate decline at $60 \mathrm{~min}$. Thus, the brief stimulation periods used in the physiological experiments described above did produce significant increases in cAMP levels in the muscle.

Next, we tested whether the cAMP levels remained clcvated over the longer stimulation periods because the SCPs were being continually released throughout the stimulation period or because the elevation of cAMP levels was persistent. B15 was stimulated for $3 \mathrm{~min}$, and both the stimulated and the control muscles were frozen at specific times following the end of the stimulation period. Maximal increases in cAMP were observed $30 \mathrm{sec}$ after stimulation, following which the cAMP levels decayed to control levels within an additional $30 \mathrm{sec}$ (Fig. 8). Therefore, the maintained elevation of cAMP levels at the end of the longer stimulation periods is likely to reflect continual release of the SCPs throughout the length of stimulation. The decay of elevated cAMP levels was much more rapid than the decay of the B15-induced modulation of B16-evoked contractions. However, the phenomenon of second-messenger-mediated effects being far more persistent than the increases in 
second-messenger levels themselves has been commonly observed in Aplysia and other preparations (Schwartz and Greenberg, 1987).

\section{Are the effects of B15 on B16-evoked contractions physiological?}

Aplysia californica is found in coastal waters that range in temperature from below $15^{\circ} \mathrm{C}$ to above $20^{\circ} \mathrm{C}$ during the year (Reish, 1961). In vivo recordings of EJPs from 15 muscles in feeding animals indicate that, at $16^{\circ} \mathrm{C}, \mathrm{B} 15$ fires at up to $12 \mathrm{~Hz}$ for 3.5 sec with 3.5-sec interburst intervals (Cropper et al., 1990). At $22^{\circ} \mathrm{C}$, however, the threshold for significant elevation of cAMP was around $15 \mathrm{~Hz}$ with 4 -sec bursts and 3-sec intervals (Whim and Lloyd, 1989). Therefore, the question arises as to the physiological relevance of the ability of $\mathrm{B} 15$ stimulation to modulate both muscle cAMP levels and neuromuscular efficacy. Because the in vivo recordings and biochemical experiments were conducted at different temperatures, we examined whether the threshold for the effects of B15 stimulation on cAMP levels varied with temperature. The results demonstrate that, whereas stimulation of B15 at $15 \mathrm{~Hz}$ (4-sec bursts, 3-sec intervals) was necessary to increase cAMP levels at $22^{\circ} \mathrm{C}$, the threshold frequency dropped to about $10 \mathrm{~Hz}$ at $16^{\circ} \mathrm{C}$ (Fig. 9). This effect of temperature on the ability of $\mathrm{B} 15$ stimulation to elevate muscle cAMP levels could have a presynaptic or postsynaptic locus (or a combination of both). If the effect was predominantly postsynaptic, the elevation of muscle cAMP levels produced by exogenous SCPs should be greater at $16^{\circ} \mathrm{C}$ than at $22^{\circ} \mathrm{C}$. However, a comparison between the effects of $10^{-7} \mathrm{M} \mathrm{SCP}_{\mathrm{B}}$ on the cAMP levels of muscle segments indicated no significant difference between the elevation at $16^{\circ} \mathrm{C}(57 \pm 26$-fold increase, $\mathrm{SEM} ; n=5)$ and $22^{\circ} \mathrm{C}(62 \pm 20$-fold increase, $n=5)$. These results indicate that the effects of temperature are likely to be mainly presynaptic, presumably an increase in the release of the SCPs from B15 terminals. In support of this hypothesis is the observation that the B15-evoked facilitated EJP increased in amplitude as the temperature was lowered. B15-evoked EJPs were recorded intracellularly in muscle fibers at $16^{\circ} \mathrm{C}$ and $22^{\circ} \mathrm{C}$. The amplitude of single EJPs did not differ at the 2 temperatures; however, facilitated EJPs were $63 \pm 44 \%$ larger at the lower temperature (SEM; 3 rd EJP in a $5-\mathrm{Hz}$ burst; 5 fibers at each temperature in each of 4 preparations). The effects on facilitated EJPs might be particularly important because our previous results suggest that release of the SCPs only occurs under conditions in which cholinergic release is significantly facilitated (Whim and Lloyd, 1989). We also investigated the effectiveness of B15 stimulation (12.5 Hz for $4 \mathrm{sec}$ with 3-scc interburst interval) in modulating B16-evoked contractions at the 2 temperatures. We had observed that, when the muscle was held at the same temperature throughout the experiment, the first tetanic stimulation of B15 always produced a larger potentiation than in subsequent trials. To control for this, the effects of $\mathrm{B} 15$ stimulation were monitored in 2 experiments, first at $16^{\circ} \mathrm{C}$, then at $22^{\circ} \mathrm{C}$, and in another 2 experiments in the reverse order. In every case, B15 stimulation produced a greater potentiation at the lower temperature, and, as expected, the potentiation was more pronounced when the first stimulation was made at $16^{\circ} \mathrm{C}(252 \%$, $203 \%$ larger potentiation at $16^{\circ} \mathrm{C}$ compared to $22^{\circ} \mathrm{C}$ ) than when the first stimulation was done at $22^{\circ} \mathrm{C}(105 \%, 179 \%$ larger at $16^{\circ} \mathrm{C}$ compared to $22^{\circ} \mathrm{C}$ ).

Because our results indicated that stimulation of B15 at physiological frequencies affected muscle cAMP levels, we investi-

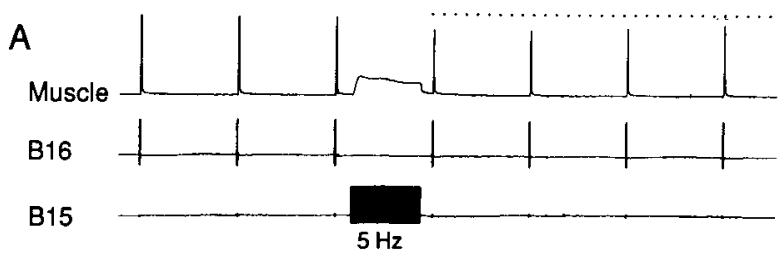

B

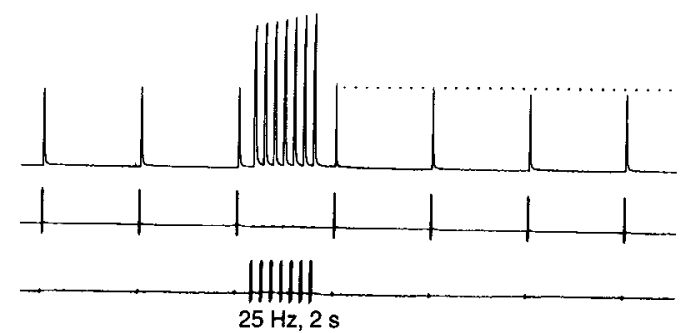

C

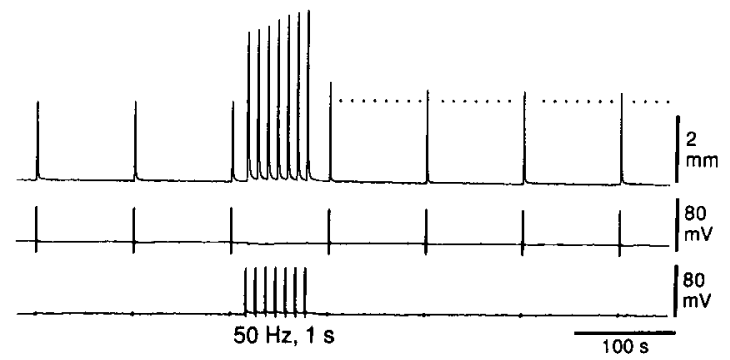

Figure 5. Modulation of B16-evoked muscle contractions by B 15 is sensitive to pattern of stimulation. $A, 15$ muscle contractions were evoked by stimulation of B16 at $20 \mathrm{~Hz}$ for $1 \mathrm{sec}$ every $100 \mathrm{sec}$. B15 was stimulated for $70 \mathrm{sec}$ at a tonic $5 \mathrm{~Hz}$ (evoking a maintained contracture of the muscle). The following B16-induced muscle contractions exhibit a reduced amplitude that slowly recovers to control levels. $B$, Effects of stimulating B15 at $25 \mathrm{~Hz}$ for $2 \mathrm{sec}$ with 8-sec intervals for $70 \mathrm{sec}$. No long-lasting modulation of the $\mathrm{I} 5$ muscle contractions was observed. $C$, Stimulation of B15 at a frequency of $50 \mathrm{~Hz}$ for $1 \mathrm{sec}$ with 9-sec intervals for $70 \mathrm{sec}$ resulted in a long-lasting potentiation of B16-evoked muscle contractions. Note that the 3 B 15 -stimulation paradigms evoked the same total number of spikes. The dotted lines indicate the basal amplitude of the B16-evoked contractions prior to B15 stimulation.

gated whether stimulation of B15 also modulated muscle contractions induced by stimulation of B16 over a range of frequencies. This was indeed the case. Typically, the lower the B16 frequency and hence the smaller the contraction, the larger the proportional increase produced by B 15 stimulation (Fig. 10). However, B15 stimulation potentiated B16-evoked contractions at all B16 frequencies tested $(5-25 \mathrm{~Hz})$. In vivo recordings indicate that, during feeding, B16 fires at $15-20 \mathrm{~Hz}$ (Cropper et al., 1990). Therefore, the modulatory actions of B15 on B16evoked contractions function in the physiological range of firing frequencies for both motor neurons.

\section{Stimulation of B16 also modulates B15-evoked muscle contractions}

If the modulation of $\mathrm{I} 5$ contractions described above is related to the release of the SCPs from B15, then the release of myomodulin from B16, a peptide known to have many of the same actions as the SCPs on muscle contractions (c.g., see Fig. 2), might similarly affect the amplitude and relaxation rate of muscle contractions. Therefore, we investigated the effects of stimulating B 16 on muscle contractions produced by stimulating B15 using the same protocol as used for the reverse experiments 
Figure 6. Modulation of B16-evoked muscle contractions by stimulation of B15 was not dependent on cholinergic neuromuscular transmission. $A, \mathrm{~B} 16$ was stimulated at $20 \mathrm{~Hz}$ for $1 \mathrm{sec}$ with $100-\mathrm{sec}$ intervals to produce control muscle contractions. Subsequent introduction of $10^{-4} \mathrm{M}$ hexamethonium, a cholinergic antagonist, into the bath blocked the B16-evoked muscle contractions (Hexamethonium). Washing produced recovery. $B$, The same paradigm as in $A$ was repeated, except that once the B16-evoked contractions were substantially blocked, B15 was stimulated at $20 \mathrm{~Hz}$ for $4 \mathrm{sec}$ with intervals of $6 \mathrm{sec}$ for $45 \mathrm{sec}$ (Hexamethonium). The brief contraction produced by the first B15 burst was consistently observed and suggests that a component of the inhibition by hexamethonium was dependent on activity in B15. The B16-evoked muscle contractions that returned during the washout of the hexamethonium from the bath were increased in amplitude and relaxation rate when compared to the control contractions produced prior to $\mathrm{B} 15$ stimulation (cf. $A, B$ ). A constant flow rate was maintained throughout to ensure that the addition of hexamethonium and its removal occurred at identical rates in both parts of the experiment. $C$, Combined data from 3 separate preparations using the paradigm described above. Solid bars indicate the amplitude of B16-evoked muscle contractions that return after the washout of hexamethonium from the bath. Open bars indicate a similar recovery of B16evoked muscle contractions after stimulation of B15 (arrow). Each interval is $100 \mathrm{sec}$ in duration and includes $2 \mathrm{~B} 16$ evoked contractions. Values are mean \pm SEM.

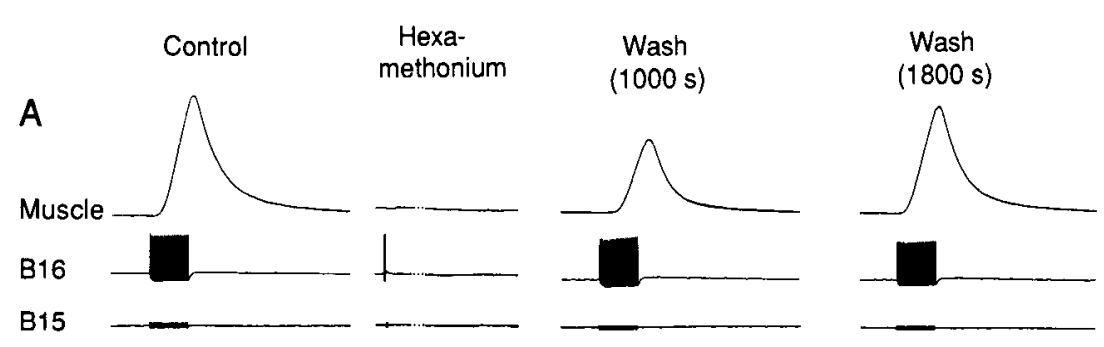

B
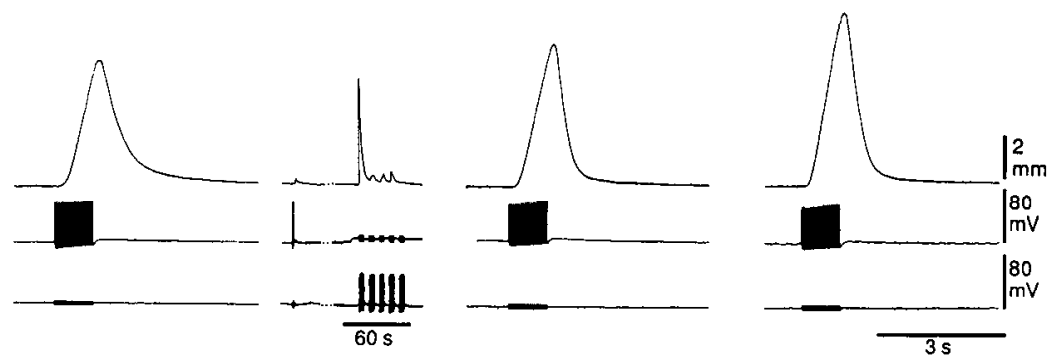

C

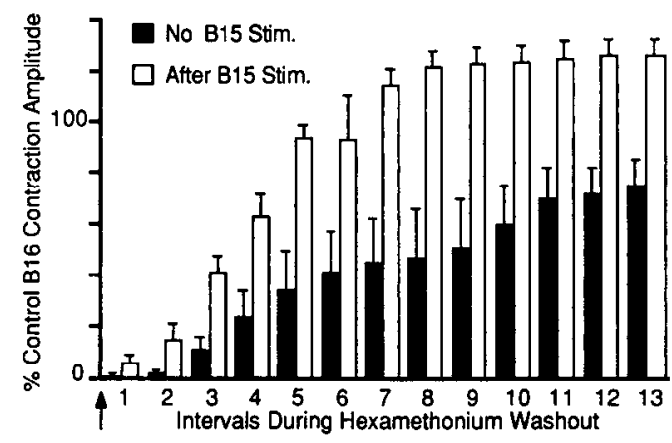

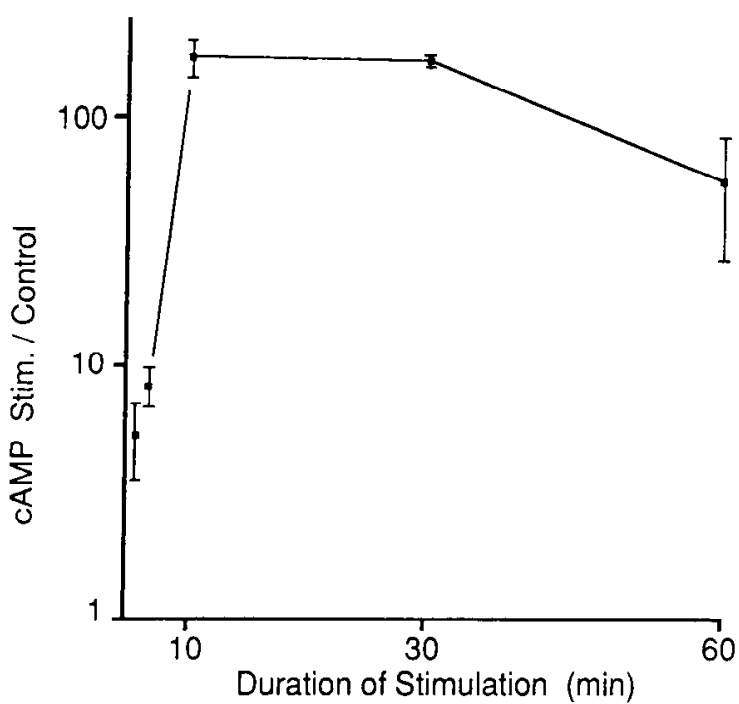

Figure 7. Effect of the duration of B15 stimulation on cAMP levels in 15 muscles. B15 was stimulated at $25 \mathrm{~Hz}$ in 4-sec bursts with 6-sec intervals for varying durations. cAMP normalized to protein of stimulated muscle (Stim.) was compared to that of the unstimulated contralateral control muscle from the same animal. The first 2 points represent durations of 1 and $3 \mathrm{~min}$, respectively. Values are mean $\pm \mathrm{SEM}$; $n=3$.
(Fig. 11). An increase in contraction amplitude and muscle relaxation rate was observed following B 16 stimulation. This suggests that myomodulin was released from B 16 terminals, though it should be emphasized that, in contrast to the SCPs, we have no independent evidence for this release.

\section{Homosynaptic facilitation of B16- and B15-evoked contractions}

Although we were mainly concerned with the heterosynaptic facilitation of B16-induced muscle activity, the ability of B15 and $\mathrm{B} 16$ stimulation to facilitate their own contractions was also examined. Stimulation of B 15 to fire bursts every 100 sec evoked reproducible 15 contractions that were potentiated when an additional series of B15 bursts was interposed (Fig. 12). This potentiation consisted of an increase in contraction amplitude and muscle relaxation rate. Using an identical stimulation paradigm, stimulation of B16 was found to produce a similar homosynaptic facilitation of B16-evoked contractions (data not shown). Although these observations are consistent with the actions of released peptides, presynaptic effects such as posttetanic potentiation of compound EJPs must also be involved (Cohen et al., 1978). 


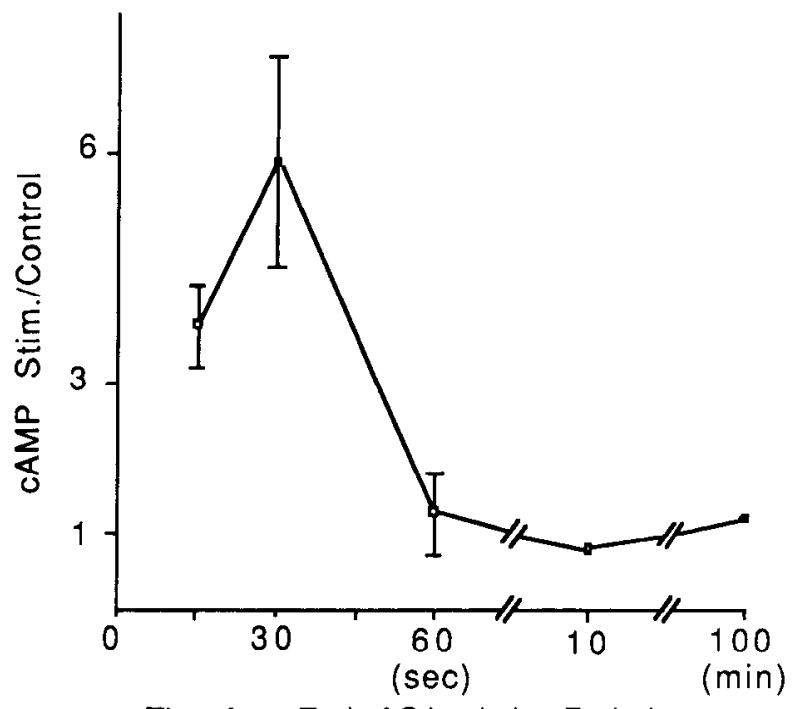

Time from End of Stimulation Period

Figure 8. Time course of decay of B15-induced elevation of 15 muscle cAMP levels. B15 was stimulated at $25 \mathrm{~Hz}$ in 4-sec bursts with 6-sec intervals for $3 \mathrm{~min}$. Stimulated and control muscles were frozen and extracted after specific times following the end of the stimulation period. cAMP normalized to protein of stimulated muscle (Stim.) was compared to that of the unstimulated contralateral control muscle from the same animal. Values are mean $\pm \operatorname{SEM}(n=3)$, except for 10 and $100 \mathrm{~min}$, which are mean $(n=2)$.

\section{Discussion}

The present study describes a novel, long-lasting modulation of the actions of one motor neuron (B16) by another (B15). Specifically, stimulation of B15 at high frequency or at lower frequency in prolonged bursts potentiates the amplitude and relaxation rate of muscle contractions evoked by stimulation of B16. The magnitude of this potentiation is not simply a function of the number of B15 spikes but is contingent on the pattern of stimulation. There could be several explanations for this modulation. The possibility that it is due to the B15-induced muscle contractions themselves appears unlikely, because inhibiting cholinergic transmission during the period of B15 activity does not prevent the subsequent potentiation of the muscle contraction amplitude or relaxation rate. In addition, modulation of muscle contractions occurs without a measurable increase in the B16-evoked compound EJPs, indicating that the effect is likely to be a modulation of the excitation-contraction coupling mechanism. Thereforc, our favorcd cxplanation for the obscrved modulation is that, under specific stimulation conditions, the SCPs are released from the terminals of B15 and act postsynaptically to produce a cAMP-mediated modulation of muscle contractions. A number of lines of evidence support this hypothesis. First, the SCPs are synthesized by B 15 and transported to the I5 muscle, where they are localized to dense core vesicles within varicosities (Lloyd et al., 1984; Cropper et al., 1988; Lloyd, 1988). Second, the actions of exogenously applied SCPs are very similar to the effects produced by B15 stimulation. Both increase muscle cAMP levels and potentiate the amplitude and rate of relaxation of contractions. Previous studies have implicated cAMP in the modulation of 15 contractions by the SCPs and serotonin. For example, application of CAMP analogs, the phosphodiesterase inhibitor RO 20-1724, or the adenylyl cyclase activator forskolin all enhance the amplitude and in-

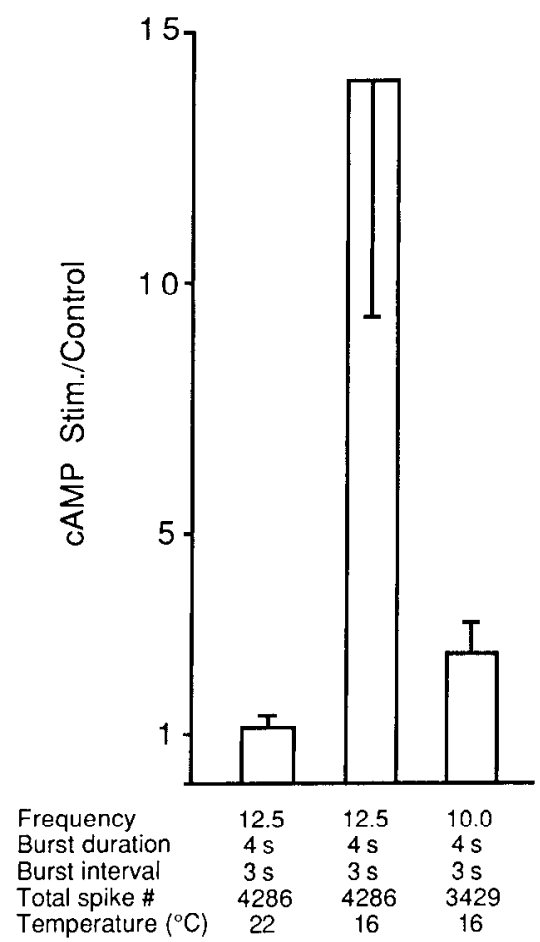

Figure 9. Temperature dependence of B15-induced elevation of cAMP levels in I5 muscles. cAMP normalized to protein of stimulated muscle (Stim.) was compared to that of the unstimulated contralateral control muscle from the same animal. Burst interval refers to the period from the end of a burst to the beginning of the next burst. Values are mean $\pm \operatorname{SEM}(n=4)$.

crease the rate of relaxation of motor-neuron-driven contractions (Weiss et al., 1979; Lloyd et al., 1984; M. D. Whim and P. E. Lloyd, unpublished observations). The B15 stimulation parameters necessary to evoke the modulation are very similar to those required for release of the SCPs (Whim and Lloyd, 1989). Therefore, stimulating B15 at a tonic $5 \mathrm{~Hz}$, or in 2-sec bursts at 8-sec intervals, produces no release of the SCPs and no sustained enhancement of muscle contractions, whereas stimulating B15 at $50 \mathrm{~Hz}$ for $1 \mathrm{sec}$ with 9-sec intervals produces both release of the SCPs and a long-lasting potentiation of contractions. It should be noted, however, that the B15 stimulation frequencies required for threshold modulatory effects on contractions are likely to be lower than those required to produce measurable increases in muscle cAMP levels. Finally, the elevation of muscle cAMP lcvels occurs rapidly enough to account for the modulation of the B16-evoked contractions observed following B15 stimulation. From these observations, it seems reasonable to conclude that a significant component of the modulation of muscle contractions is due to the stimulation-dependent release of the SCPs from B15.

Because the elevation of cAMP by released SCPs does not desensitize in under $30 \mathrm{~min}$, the relatively rapid decline of the B15-stimulated cAMP levels places an upper limit of about 60 sec, during which time released SCPs remain available to bind to their receptors. Presumably, this reflects the period of time that active SCPs remain in the synaptic cleft. Consistent with this interpretation, our previous results comparing the amounts of released SCPs to their effects on muscle cAMP levels suggested that the SCPs are released into a very small volume (Whim and Lloyd, 1989). 
Figure 10. Modulation of B16-evoked muscle contractions by stimulation of B15. B16 was stimulated for $1 \mathrm{sec}$ at frequencies of 10,15 , and $20 \mathrm{~Hz}$ (bottom trace), with an interburst interval of $100 \mathrm{sec}$ to produce a family of contractions (Before B15 Stim). B15 was then stimulated at $25 \mathrm{~Hz}$ with 4-sec bursts and 6-sec interburst intervals for $60 \mathrm{sec}$ (Inset: upper trace, contractions; lower trace, B15 recording). Note the progressive increase in muscle relaxation rate that occurred during B 15 stimulation. Following B15 stimulation, B16 was again stimulated at a frequency of 10,15 , and $20 \mathrm{~Hz}$ (After B15 Stim.). The resulting muscle contractions were now increased both in amplitude and relaxation rate.

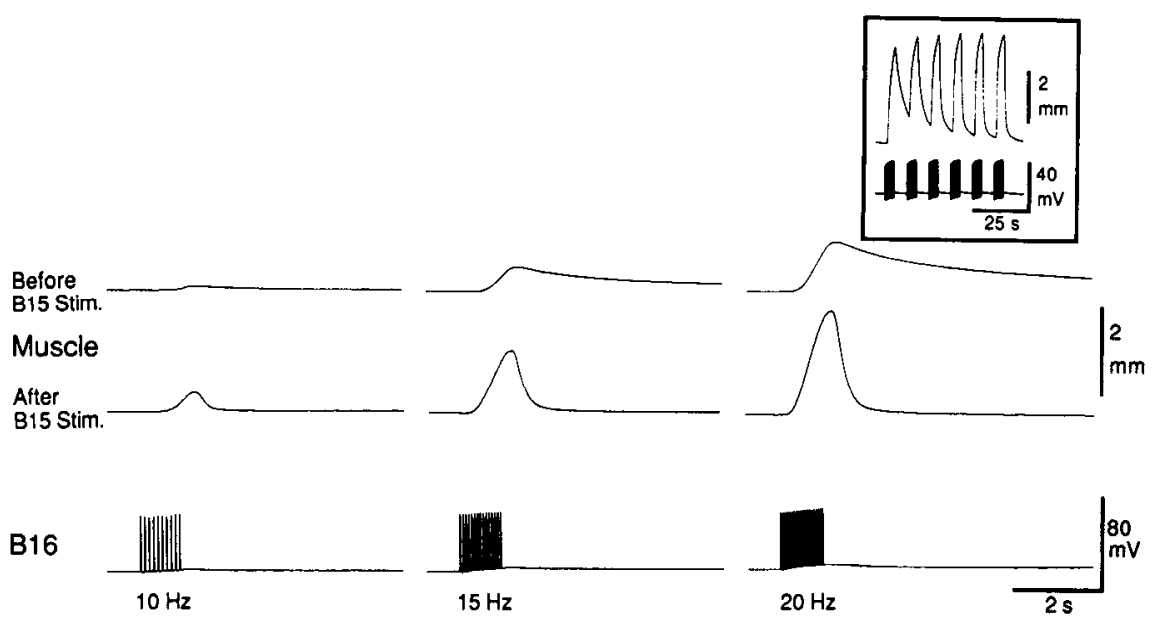

Clearly, it is of interest to know whether the B15-induced modulation of B16-evoked contractions is of physiological importance. The sharp temperature dependence of the effect of B 15 on muscle cAMP levels indicates that the apparent threshold of B 15 stimulation required to affect 15 muscle cAMP levels is as low as $10 \mathrm{~Hz}$ at $16^{\circ} \mathrm{C}$. We observed that the ability of $\mathrm{B} 15$ to potentiate B16-evoked contractions showed a similar temperature dependence. Furthermore, B15 stimulation can modulate $\mathrm{I} 5$ contractions that are produced by a wide range of B16 firing frequencies. These stimulation paradigms are within the range in which both cells fire in vivo (Cropper et al., 1990). Given these observations, it is likely that the enhancement of the amplitude and relaxation rate of motor-neuron-driven con- tractions observed upon stimulation of B15 would occur in the feeding animal.

The modulation of 15 contractions by motor neurons in the feeding animal are unlikely to be straightforward heterosynaptic effects. For example, both $\mathrm{B} 15$ and $\mathrm{B} 16$ have the ability to homosynaptically modulate their own contractions, as well as to heterosynaptically modulate the contractions of each other. It is possible that this modulation may in part underlie the potentiation of muscle contractions that is observed when the interval between successive motor neuron bursts is decreased (Cohen et al., 1978). Furthermore, the existence of modulatory capability intrinsic to the motor neurons may provide an explanation for results from studies in which the serotonergic
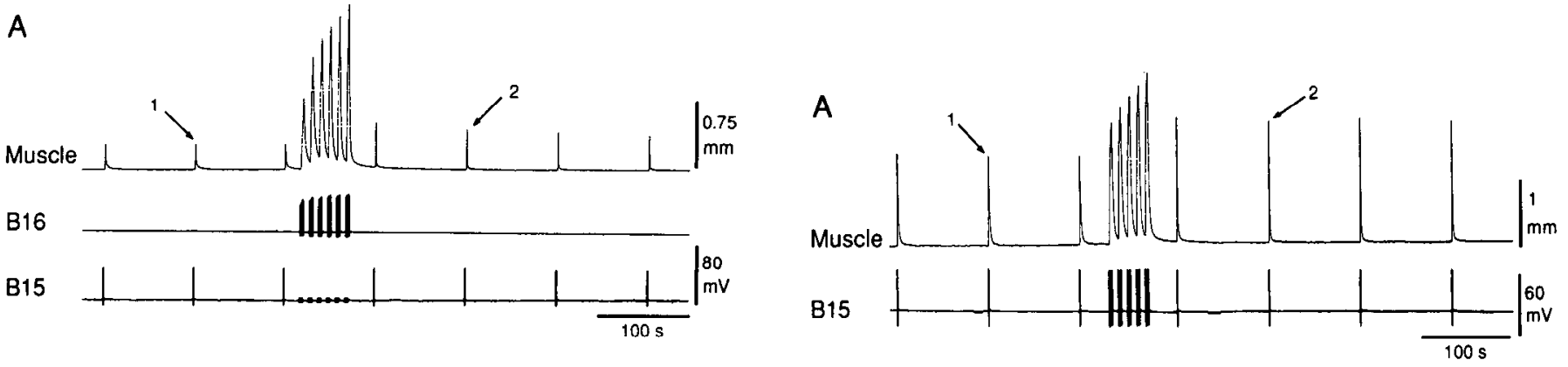

B

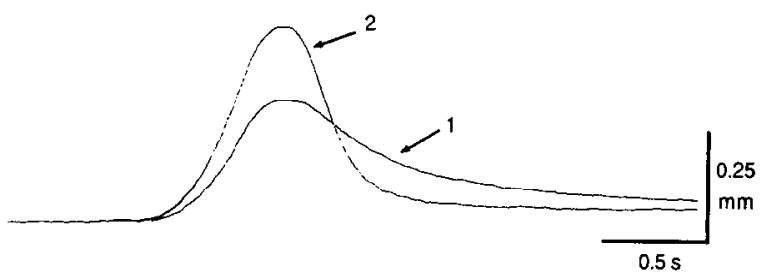

B

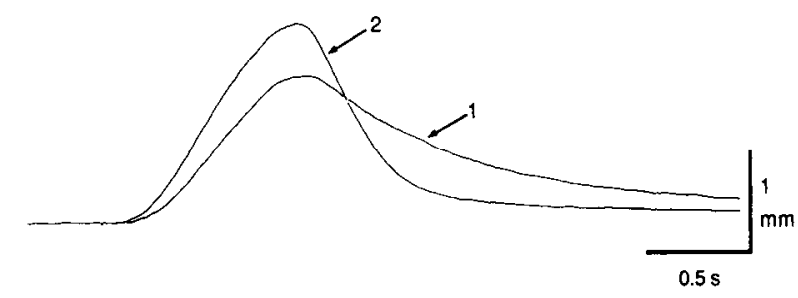

Figure 11. Modulation of B15-evoked I5 muscle contractions by stimulation of B16. A, Stimulation of B15 at $20 \mathrm{~Hz}$ for $1 \mathrm{sec}$ with an interburst interval of $100 \mathrm{sec}$ elicited reproducible 15 muscle contractions. A period of B16 stimulation ( $25 \mathrm{~Hz}$ for 4-sec bursts with an interburst interval of $6 \mathrm{sec}$ for $60 \mathrm{sec}$ ) was then interposed between successive B15 bursts. The subsequent I5 muscle contractions evoked by stimulation of B15 was increased in amplitude. $B$, Examination of B15-evoked muscle contractions at a faster chart speed before (1) and after (2) B16 stimulation illustrates that there was also an increase in the muscle relaxation rate.

Figure 12. Homosynaptic modulation of B15-evoked muscle contractions. $A$, 15 muscle contractions evoked by stimulation of $\mathrm{B} 15$ ( 20 $\mathrm{Hz}$ for $1 \mathrm{sec}$ with an interburst interval of $100 \mathrm{sec}$ ) were increased in amplitude after an interposed period of prolonged stimulation of $\mathrm{B} 15$ ( $15 \mathrm{~Hz}$ for $4 \mathrm{sec}$ with 6 -sec interburst intervals for $45 \mathrm{sec}$ ). $B$, Examination of 15 muscle contractions before $(I)$ and after (2) the interposed B15 bursts illustrates that there was an increase in muscle relaxation rate. 
metacerebral cells (MCCs) were selectively lesioned with intracellular injections of proteolytic enzymes (Rosen et al., 1983, 1989). Stimulation of the MCCs or application of serotonin to I5 muscles modulates contractions in a manner very similar to the SCPs (Weiss et al., 1978; Lloyd et al., 1984). Therefore, if the MCCs were the primary source of modulation, one would expect that lesioning the MCCs would produce a pronounced effect on bite magnitude; however, only a small effect was observed. This presumably reflects the fact the MCCs provide only one component of the total modulatory input to I5 and other buccal muscles.

Coexistence of modulatory neuropeptides with conventional transmitters has been reported previously in invertebrates (O'Shea and Bishop, 1982; Bishop et al., 1984; Schwarz et al., 1984; Kuhlman et al., 1985; O'Shea and Schaffer, 1985), as well as in vertebrates (Gibson et al., 1984; Ch'ng et al., 1985; New and Mudge, 1986). Furthermore, in a number of instances, there is evidence that these neuropeptides may modulate several aspects of neuromuscular transmission (Adams and O'Shea, 1983; New and Mudge, 1986; Bishop et al., 1987; Mulle et al., 1988). Therefore, modulation by peptides released from motor neuron terminals may be phylogenetically widespread.

In conclusion, we believe that significant modulation of muscle contractions by peptides released from buccal motor neurons in Aplysia is most likely to occur during food-induced arousal when buccal motor neurons fire at high frequency with brief interburst intervals (Susswein et al., 1978). An interesting speculation is that the more important component of this modulation may be the increased rate of muscle relaxation, which would permit the execution of rapid repeated biting movements. Although this study was directed specifically to the I5 muscle, there are several reasons to believe similar modulation by the SC.Ps is widespread among other buccal muscles. First, the levels of the SCPs found in muscles, and the rates at which SCPs synthesized in buccal ganglia are transported to muscles, are similar for I5 and the other major buccal muscles (Lloyd, 1988; Whim and Lloyd, 1989). Second, many of the buccal muscle motor neurons synthesize and contain the SCPs (Lloyd et al., 1985, 1987b). Finally, modulation of other buccal muscles by application of exogenous SCPs has been observed (Richmond et al., 1986; Lotshaw and Lloyd, 1990). Therefore, modulation of buccal muscle contractions by SCPs released from motor neurons may contribute significantly to the behavioral plasticity observed during feeding in Aplysia.

\section{References}

Abrams TW, Castellucci VF, Camardo JS, Kandel ER, Lloyd PE (1984) Two endogenous neuropeptides modulate the gill and siphon withdrawal reflex in Aplysia by presynaptic facilitation involving cAMPdependent closure of a serotonin-sensitive potassium channel. Proc Natl Acad Sci USA 81:7956-7960.

Adams ME, O'Shca M (1983) Pcptide cotransmitters at a neuromuscular junction. Science 221:286-289.

Bishop CA, Wine JJ, O'Shea M (1984) Neuropeptide proctolin in postural motor neurons of the crayfish. J Neurosci 4:2001-2009.

Bishop CA, Wine JJ, Nagy F, O'Shea MR (1987) Physiological consequences of a peptide cotransmitter in a crayfish nerve-muscle preparation. J Neurosci 7:1769-1779.

Ch'ng JL, Christofides ND, Anand P, Gibson SJ, Allen YS, Su HC, Tatemoto K, Morrison JFB, Polak JM, Bloom SR (1985) Distribution of galanin immunoreactivity in the central nervous system and the responses of galanin-containing neuronal pathways to injury. Neuroscience 16:343-354

Cohen JL, Weiss KR, Kupfermann I (1978) Motor control of buccal muscles in Aplysia. J Neurophysiol 41:157-180.
Cropper EC, Lloyd PE, Reed W, Tenenbaum R, Kupfermafn I, Weiss KR (1987a) Multiple neuropeptides in cholinergic motor neurons of Aplysia: evidence for modulation intrinsic to the motor circuit. Proc Natl Acad Sci USA 84:3486-3490.

Cropper EC, Tenenbaum R, Gawinowicz Kolks MA, Kupfermann I, Weiss KR (1987b) Myomodulin: a bioactive neuropeptide present in an identificd cholinergic buccal motor neuron of Aplysia. Proc Natl Acad Sci USA 84:5483-5486.

Cropper EC, Miller MW, Tenenbaum R, Gawinowicz Kolks MA, Kupfermann I, Weiss KR (1988) Structure and action of buccalin: a modulatory neuropeptide localized to an identified small cardioactive peptide-containing cholinergic motor neuron of Aplysia californica. Proc Natl Acad Sci USA 85:6177-6181.

Cropper EC, Price D, Tenenbaum R, Kupfermann I, Weiss KR (1990) Release of peptide cotransmitters from a cholinergic neuron under physiological conditions. Proc Natl Acad Sci USA 87:933-937.

Gibson SJ, Polak JM, Bloom SR, Sabate IM, Muldberry PM, Ghatel MA, McGregor GP, Morrison JFB, Kelly JS, Evans RM, Rosenfeld MG (1984) Calcitonin gene-related peptide immunoreactivity in the spinal cord of man and eight other species. J Neurosci 4:3101-3111.

Howells HH (1942) The structure and function of the alimentary canal of Aplysia punctata. Q J Microsc Sci 83:357-397.

Kreiner T, Sossin W, Scheller RH (1986) Localization of Aplysia neurosecretory peptides to multiple populations of dense core vesicles. $\mathbf{J}$ Cell Biol 102:769-782.

Kuhlman JR, Li C, Calabrese RL (1985) FMRF-amide-like substances in the leech. I. Immunocytological localization. J Neurosci 5:23012309.

Kupfermann I, Cropper EC, Miller MW, Alevizos A, Tenenbaum R, Weiss KR (1988) Buccalin: distribution of immunoreactivity in the Aplysia nervous system and biochemical localization to motor neuron B16. Soc Neurosci Abstr 14:177.

Lloyd PE (1986) The small cardioactive peptides: a class of modulatory neuropeptides in Aplysia. Trends Neurosci 9:428-431.

Lloyd PE (1988) Fast axonal transport of modulatory ncuropeptides from central ganglia to components of the feeding system in Aplysia. J Neurosci 8:3507-3514.

Lloyd PE, Kupfermann I, Weiss KR (1984) Evidence for parallel actions of a molluscan peptide $\left(\mathrm{SCP}_{\mathrm{B}}\right)$ and serotonin in mediating arousal in Aplysia. Proc Natl Acad Sci USA 81:2934-2937.

Lloyd PE, Mahon AC, Kupfermann I, Cohen JL, Scheller RH, Weiss $\mathrm{KR}$ (1985) Biochemical and immunocytological localization of molluscan small cardioactive peptides (SCPs) in the nervous system of Aplysia californica. J Neurosci 5:1851-1861.

Lloyd PE, Schacher S, Kupfermann I, Weiss KR (1986) Calciumdependent release of neuropeptides during intracellular stimulation of single identified Aplysia neurons in culture. Proc Natl Acad Sci USA 83:9794-9798.

Lloyd PE, Kupfermann I, Weiss KR (1987a) The sequence of small cardioactive peptide A: a second member of a class of neuropeptides in Aplysia. Peptides 8:179-184.

Lloyd PE, Frankfurt M, Stevens P, Kupfermann I, Weiss KR (1987b) Biochemical and immunocytological localization of the neuropeptides FMRFamide, $\mathrm{SCP}_{\mathrm{A}}, \mathrm{SCP}_{\mathrm{B}}$, to neurons involved in the regulation of feeding in Aplysia. J Neurosci 7:1123-1132.

Lloyd PE, Kupfermann I, Weiss KR (1988) Central peptidergic neurons regulate gut motlity in Aplysia. J Neurophysiol 59:1613-1626.

Lotshaw DP, Lloyd PE (1990) Peptidergic and serotonergic facilitation of a neuromuscular synapse in Aplysia. Brain Res., in press.

Mahon AC, Lloyd PE, Weiss KR, Kupfermann I, Scheller RH (1985) The small cardioactive peptides A and B of Aplysia are derived from a common precursor molecule. Proc Natl Acad Sci USA 82:39253929.

Morris HR, Panico M, Karplus A, Lloyd PE, Riniker B (1982) Elucidation by FAB-MS of the structure of a new cardioactive peptide from Aplysia. Nature 300:643-645.

Mulle C, Benoit P, Pinset C, Roa M, Changeux J-P (1988) Calcitonin gene-related peptide enhances the rate of desensitization of the nicotinic acetylcholine receptor in cultured mouse muscle cells. Proc Natl Acad Sci USA 85:5728-5732.

New HV, Mudge AW (1986) Calcitonin gene-related peptide regulates muscle acetylcholine receptor synthesis. Nature 323:809-811.

O'Shea M, Bishop CA (1982) Neuropeptide proctolin associated with an identified skeletal motoneuron. J Neurosci 4:521-529.

O'Shea M, Schaffer M (1985) Neuropeptide function: the invertebrate contribution. Annu Rev Neurosci 8:171-198. 
Reed W, Weiss KR, Lloyd PE, Kupfermann I, Chen M, Bailey $\mathrm{CH}$ (1988) Association of neuroactive peptides with the protein secretory pathway in identified neurons of Aplysia californica: immunolocalization of $\mathrm{SCP}_{\mathrm{A}}$ and $\mathrm{SCP}_{\mathrm{g}}$ to the contents of dense-core vesicles and the trans face of the Golgi apparatus. J Comp Neurol 272:358-369.

Reish DJ (1961) A study of benthic fauna in a recently constructed boat harbor in southern California. Ecology 42:84-91.

Richmond JE, Bulloch AGM, Lukowiak K (1986) Peptidergic modulation of a neuromuscular junction in Aplysia: bioactivity and immunocytochemistry. Brain Res 370:159-164.

Rosen SC, Kupfermann I, Goldstein RS, Weiss KR (1983) Lesion of serotonergic modulatory neuron produces a specific deficit in feeding behavior. Brain Res 260:151-155.

Rosen SC, Weiss KR, Goldstein RS, Kupfermann I (1989) The role of a modulatory neuron in feeding and satiation in Aplysia: effects of lesioning of the serotonergic metacerebral cells. J Neurosci 9:15621578.

Schwartz JH, Greenberg SM (1987) Molecular mechanisms for mem- ory: second-messenger induced modifications of protein kinases in nerve cells. Ann Rev Neurosci 10:459-476.

Schwarz TL, Lee GMH, Siwicki KK, Standaert DG, Kravitz EA (1984) Proctolin in the lobster: the distribution, release, and characterization of a likely neurohormone. J Neurosci 4:1300-1311.

Susswein A, Weiss KR, Kupfermann I (1978) The effects of food arousal on the latency of biting in Aplysia. J Comp Physiol 123:31-41.

Weiss KR, Cohen JL, Kupfermann I (1978) Modulatory control of buccal musculature by a serotonergic neuron (metacerebral cell) in Aplysia. J Neurophysiol 41:181-203.

Weiss KR, Mandelbaum DR, Schonberg M, Kupfermann I (1979) Modulation of buccal muscle contractibility by serotonergic metacerebral cells in Aplysia: evidence for a role of cyclic adenosine monophosphate. J Neurophysiol 42:791-803.

Whim MD, Lloyd PE (1989) Frequency-dependent release of peptide cotransmitters from identified cholinergic motor neurons in Aplysia. Proc Natl Acad Sci USA 86:9034-9038. 